\title{
One-loop divergences in non-Abelian supersymmetric theories regularized by BRST-invariant version of the higher derivative regularization
}

\author{
S.S. Aleshin, A.E. Kazantsev, M.B. Skoptsov and K.V. Stepanyantz \\ Moscow State University, Physical Faculty, Department of Theoretical Physics, \\ Moscow, 119991 Russia \\ E-mail: aless2001@mail.ru, kazancev@physics.msu.ru, skoptsov23@ya.ru, \\ stepan@m9com.ru
}

ABSTRACT: We consider a general non-Abelian renormalizable $\mathcal{N}=1$ supersymmetric gauge theory, regularized by higher covariant derivatives without breaking the BRST invariance, and calculate one-loop divergences for a general form of higher derivative regulator and of the gauge fixing term. It is demonstrated that the momentum integrals giving the one-loop $\beta$-function are integrals of double total derivatives independently of a particular choice of the higher derivative term. Evaluating them we reproduce the well-known result for the one-loop $\beta$-function. Also we find that the three-point ghost vertices with a single line of the quantum gauge superfield are not renormalized in the considered approximation.

KEYwords: Renormalization Regularization and Renormalons, Supersymmetric gauge theory, Gauge Symmetry

ARXIV EPRINT: 1603.04347 


\section{Contents}

1 Introduction 1

2 The BRST-invariant higher covariant derivative regularization for $\mathcal{N}=1$ supersymmetric gauge theories 3

2.1 Action of the considered theory 3

2.2 The background field method 4

2.3 The higher covariant derivative regularization 5

2.4 Renormalization 8

$\begin{array}{lll}2.5 & \text { RG functions } & 10\end{array}$

3 The RG functions in the one-loop approximation 10

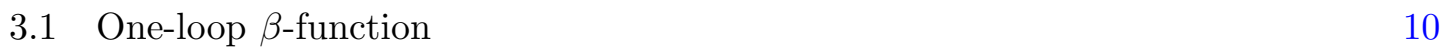

$\begin{array}{ll}3.2 & \text { One-loop anomalous dimension of the matter superfields } \\ & 12\end{array}$

$\begin{array}{lll}3.3 & \text { One-loop renormalization of the quantum gauge superfield } & 13\end{array}$

$\begin{array}{ll}3.4 & \text { One-loop renormalization of the Faddeev-Popov ghosts } \\ \end{array}$

4 Conclusion $\quad 15$

\section{Introduction}

The well-known non-renormalization theorem [1] states that in $\mathcal{N}=1$ supersymmetric gauge theories the superpotential does not receive divergent quantum corrections. Moreover, there is one more interesting feature of quantum corrections in these theories. Namely, the renormalization of the coupling constant is related to the renormalization of the matter superfields by the so-called exact NSVZ $\beta$-function [2-7]. For the general $\mathcal{N}=1$ supersymmetric Yang-Mills (SYM) theory with matter it is written as

$$
\beta(\alpha, \lambda)=-\frac{\alpha^{2}\left(3 C_{2}-T(R)+C(R)_{i}{ }^{j}\left(\gamma_{\phi}\right)_{j}{ }^{i}(\alpha, \lambda) / r\right)}{2 \pi\left(1-C_{2} \alpha / 2 \pi\right)},
$$

where $\left(\gamma_{\phi}\right)_{j}{ }^{i}$ denotes the anomalous dimension of the chiral matter superfields and the following notation is used:

$$
\begin{aligned}
\operatorname{tr}\left(T^{A} T^{B}\right) & \equiv T(R) \delta^{A B} ; & \left(T^{A}\right)_{i}{ }^{k}\left(T^{A}\right)_{k}{ }^{j} & \equiv C(R)_{i}{ }^{j} ; \\
f^{A C D} f^{B C D} & \equiv C_{2} \delta^{A B} ; & r & \equiv \delta_{A A} .
\end{aligned}
$$

(It is assumed that the generators of the fundamental representation $t^{A}$ are normalized by the condition $\operatorname{tr}\left(t^{A} t^{B}\right)=\delta^{A B} / 2$.)

In the early papers the NSVZ $\beta$-function was obtained from general arguments, such as the structure of instanton contributions to the effective action $[2,8]$, anomalies $[3,5,9]$, 
non-renormalization of the topological term [10]. However, it was also necessary to construct the subtraction scheme in which the NSVZ $\beta$-function is valid. The explicit loop calculations [11-15] (see [16] for a review) made with the dimensional reduction [17] in the $\overline{\mathrm{DR}}$-scheme agree with the NSVZ $\beta$-function only after a special finite renormalization which should be constructed in each order $[12,18]$. Up to now, there is no general prescription how to do it in an arbitrary order. However, a possibility of making this finite renormalization is nontrivial [12], because from the general equation which describes how the NSVZ expression is changed under a finite renormalization [19, 20] one can derive some scheme independent consequences of the NSVZ relation [20, 21].

At least in the Abelian case, the NSVZ scheme can be naturally constructed if the supersymmetric theories are regularized by higher covariant derivatives [22, 23]. This regularization is mathematically consistent unlike the dimensional reduction [24]. (Removing the inconsistencies of the dimensional reduction leads to the loss of the explicit supersymmetry [25], which can be in this case broken by higher order quantum corrections [26-28].) The higher covariant derivative regularization can be formulated in the explicitly $\mathcal{N}=1$ supersymmetric way $[29,30]$, so that it does not break supersymmetry. It can be also used for regularization of $\mathcal{N}=2$ supersymmetric theories [31-33].

With the higher covariant derivative regularization the NSVZ relation was derived for the Abelian supersymmetric theories in all orders for the renormalization group (RG) functions defined in terms of the bare coupling constant [34,35] (which are scheme-independent for a fixed regularization [36]). The RG functions defined in the standard way in terms of the renormalized coupling constant [37] satisfy the NSVZ relation only in the NSVZ scheme which in this case can be constructed in all orders by imposing simple boundary conditions on the renormalization constants $[20,36]$. Thus, the NSVZ scheme can be easily constructed with the Slavnov higher derivative regularization. The main feature of quantum corrections, which allows to do this, is the factorization of integrals for the $\beta$-function (defined in terms of the bare coupling constant) into integrals of (double) total derivatives in the momentum space in the limit of the vanishing external momentum [38, 39]. In the Abelian case this has been proved in all orders [34, 35] and confirmed by explicit calculations in the three-loop approximation [40]. Using a similar method it has been proved that the integrals for the Adler $D$-function [41] (defined in terms of the bare coupling constant) in $\mathcal{N}=1$ supersymmetric QCD are also integrals of double total derivatives in all orders. This feature allows to relate this function to the anomalous dimension of the matter superfields exactly in all orders $[42,43]$. This new relation is similar to the NSVZ $\beta$-function and has a similar origin.

In the non-Abelian case the calculations of the $\beta$-function with the higher covariant derivative regularization were made only in the two-loop order [44, 45], where it was demonstrated that all momentum integrals giving the $\beta$-function are integrals of total derivatives. Subsequently, the results of the papers $[44,45]$ were written in the form of integrals of double total derivatives [46-48]. However, the versions of the higher covariant derivative regularization which were used for making explicit calculations for the non-Abelian $\mathcal{N}=1$ supersymmetric theories break the BRST invariance [49, 50] (while the background gauge invariance is not broken). Then the calculations are much simpler in comparison with the 
version of the higher derivative regularization which does not break the BRST invariance. Certainly, using of non-invariant regularizations is possible (see, e.g., [51-54]), if they are supplemented by a subtraction scheme which restores the Slavnov-Taylor identities [55, 56]. However, it is much more convenient to make calculations with the invariant regularization. Moreover, the invariant regularization may be useful for the general derivation of the NSVZ relation in the non-Abelian case. That is why in the present paper we consider a more complicated version of the higher derivative regularization which does not break the BRST invariance and very general forms of the higher derivative term and of the gauge fixing term. In this case the calculations are much more complicated. That is why here we make them only in the one-loop approximation. Certainly, in the one-loop approximation the higher derivative regularization always gives the result which is in agreement with other regularizations [57]. Nevertheless, the one-loop calculations can be used for demonstrating the factorization of integrals which give the $\beta$-function into integrals of double total derivatives. Moreover, they allow to verify the method of calculations and fix some potential problems. For example, the first calculation of the quantum corrections made with the higher covariant derivative regularization for the (non-supersymmetric) Yang-Mills theory [58] gave the correct result for the one-loop $\beta$-function [59,60] only after corrections made in [61, 62]. (One-loop quantum corrections in non-supersymmetric electrodynamics with the higher derivative term were also recently investigated in [63].) There are also other subtleties in calculating quantum corrections in supersymmetric theories, see, e.g., [9, 64, 65]. One more important reason for making the one-loop calculation is that for deriving the NSVZ relation by the direct summation of supergraphs in all orders (such as in refs. [34, 35]) this approximation should be considered separately and the BRST invariant regularization is highly desirable.

This paper is organized as follows. In section 2 we regularize the $\mathcal{N}=1$ SYM theory by higher covariant derivatives without breaking the BRST invariance and construct the generating functional for the regularized theory. In section 3 we calculate the one-loop divergences for various Green functions. In particular, we demonstrate that all integrals giving the one-loop $\beta$-function are integrals of double total derivatives in the momentum space independently of the form of the higher derivative term, and ghost vertices with a single gauge line are finite.

\section{The BRST-invariant higher covariant derivative regularization for $\mathcal{N}=$ 1 supersymmetric gauge theories}

\subsection{Action of the considered theory}

In this paper we consider the general renormalizable $\mathcal{N}=1 \mathrm{SYM}$ theory. In the massless limit this theory is described by the action

$$
\begin{aligned}
S=\frac{1}{2 e_{0}^{2}} \operatorname{Retr} \int d^{4} x d^{2} \theta W^{a} W_{a}+\frac{1}{4} \int d^{4} x & d^{4} \theta \phi^{* i}\left(e^{2 V}\right)_{i}{ }^{j} \phi_{j} \\
& +\left(\frac{1}{6} \int d^{4} x d^{2} \theta \lambda_{0}^{i j k} \phi_{i} \phi_{j} \phi_{k}+\text { c.c. }\right),
\end{aligned}
$$


which is written in terms of $\mathcal{N}=1$ superfields [66, 67]. Here $e_{0}$ and $\lambda_{0}^{i j k}$ denote the bare coupling constant and the Yukawa couplings, respectively. The gauge superfield

$$
V=e_{0} V^{A} T^{A}
$$

is hermitian, so that its components, $V^{A}$, are real superfields. $\phi_{i}$ are chiral matter superfields which lie in a certain representation $R$ of the gauge group $G$. In general, this representation can be reducible. The gauge superfield strength

$$
W_{a} \equiv \frac{1}{8} \bar{D}^{2}\left(e^{-2 V} D_{a} e^{2 V}\right)=e_{0} W_{a}^{A} t^{A}
$$

is also a chiral superfield. In order to obtain a gauge invariant theory, the Yukawa couplings should satisfy the condition

$$
\lambda_{0}^{i j m}\left(T^{A}\right)_{m}{ }^{k}+\lambda_{0}^{i m k}\left(T^{A}\right)_{m}^{j}+\lambda_{0}^{m j k}\left(T^{A}\right)_{m}{ }^{i}=0 .
$$

In this case the theory (2.1) is invariant under the transformations

$$
\phi \rightarrow e^{A} \phi ; \quad e^{2 V} \rightarrow e^{-A^{+}} e^{2 V} e^{-A},
$$

where the parameter $A$ is an arbitrary chiral superfield. Under these transformations the gauge superfield strength is changed as

$$
W_{a} \rightarrow e^{A} W_{a} e^{-A}
$$

Note that in the last two equations we use the matrix notation. Explicitly writing the indexes we obtain, e.g.,

$$
\phi_{i} \rightarrow\left(e^{A}\right)_{i}{ }^{j} \phi_{j}, \quad \text { etc. }
$$

It is also convenient to introduce the superfield $\Omega$ which, by definition, satisfies the equation

$$
e^{2 V} \equiv e^{\Omega^{+}} e^{\Omega} .
$$

\subsection{The background field method}

A convenient tool for calculating quantum corrections is the background field method [6870], because it allows to obtain the explicitly gauge invariant effective action. In the supersymmetric case it is introduced by the substitution

$$
e^{\Omega} \rightarrow e^{\Omega} e^{\boldsymbol{\Omega}}, \quad \text { so that } \quad e^{2 V} \rightarrow e^{\boldsymbol{\Omega}^{+}} e^{2 V} e^{\boldsymbol{\Omega}} .
$$

Then the background gauge superfield $\boldsymbol{V}$ is defined by the equation

$$
e^{2 \boldsymbol{V}}=e^{\boldsymbol{\Omega}^{+}} e^{\boldsymbol{\Omega}} .
$$

The theory which is obtained after the substitution (2.9) is evidently invariant under the background gauge transformations

$$
e^{\Omega} \rightarrow e^{i K} e^{\Omega} e^{-A} ; \quad e^{\Omega} \rightarrow e^{\Omega} e^{-i K} ; \quad V \rightarrow e^{i K} V e^{-i K} ; \quad \phi \rightarrow e^{A} \phi,
$$


where $K$ is an arbitrary hermitian superfield and $A$ is a chiral superfield which lies in the Lie algebra of the gauge group.

Also the considered theory is invariant under the quantum gauge transformations

$$
e^{2 V} \rightarrow e^{-\mathcal{A}^{+}} e^{2 V} e^{-\mathcal{A}} ; \quad e^{\boldsymbol{\Omega}} \rightarrow e^{\boldsymbol{\Omega}} ; \quad e^{\boldsymbol{\Omega}^{+}} \rightarrow e^{\boldsymbol{\Omega}^{+}} ; \quad \phi \rightarrow e^{-\boldsymbol{\Omega}} e^{\mathcal{A}} e^{\boldsymbol{\Omega}} \phi .
$$

The parameter $\mathcal{A}$ of the quantum gauge transformations is a background chiral superfield which, by definition, satisfies the condition

$$
\bar{\nabla}_{\dot{a}} \mathcal{A}=0,
$$

where the gauge and supersymmetric background covariant derivatives are defined by

$$
\boldsymbol{\nabla}_{a}=e^{-\boldsymbol{\Omega}^{+}} D_{a} e^{\boldsymbol{\Omega}^{+}} ; \quad \bar{\nabla}_{\dot{a}}=e^{\boldsymbol{\Omega}} \bar{D}_{\dot{a}} e^{-\boldsymbol{\Omega}} .
$$

Acting on a superfield $S$ which transforms as $S \rightarrow e^{i K} S$ under the background gauge symmetry (2.11) they will have the same transformation law, e.g., $\nabla_{a} S \rightarrow e^{i K} \nabla_{a} S$.

After the substitution (2.9) the superfield strength $W_{a}$ will have the form

$$
W_{a}=\frac{1}{8} e^{-\boldsymbol{\Omega}} \bar{\nabla}^{2}\left(e^{-2 V} \nabla_{a} e^{2 V}\right) e^{\boldsymbol{\Omega}}+\boldsymbol{W}_{a},
$$

where we introduce the notation

$$
\boldsymbol{W}_{a}=\frac{1}{8} \bar{D}^{2}\left(e^{-2 \boldsymbol{V}} D_{a} e^{2 \boldsymbol{V}}\right)
$$

It is convenient to fix a gauge and introduce a regularization in such a way that the invariance (2.11) remains unbroken. Then the effective action will be also invariant under the transformations (2.11), which is very convenient for calculating RG functions.

\subsection{The higher covariant derivative regularization}

The main idea of the higher covariant derivative regularization is adding a term with higher degrees of the covariant derivatives (which we will denote by $S_{\Lambda}$ ) to the classical action. Certainly, such a term is not uniquely defined. There are a lot of options for choosing it. Here, for definiteness, we will use the following expression:

$$
\begin{aligned}
S_{\Lambda}= & \frac{1}{2 e_{0}^{2}} \operatorname{Re} \operatorname{tr} \int d^{4} x d^{2} \theta e^{\Omega} e^{\Omega} W^{a} e^{-\boldsymbol{\Omega}} e^{-\Omega}\left[R\left(-\frac{\bar{\nabla}^{2} \nabla^{2}}{16 \Lambda^{2}}\right)-1\right]_{A d j} e^{\Omega} e^{\Omega} W_{a} e^{-\boldsymbol{\Omega}} e^{-\Omega} \\
& +\frac{1}{4} \int d^{4} x d^{4} \theta \phi^{+} e^{\boldsymbol{\Omega}^{+}} e^{\Omega^{+}}\left[F\left(-\frac{\bar{\nabla}^{2} \nabla^{2}}{16 \Lambda^{2}}\right)-1\right] e^{\Omega} e^{\boldsymbol{\Omega}} \phi
\end{aligned}
$$

where the gauge and supersymmetric covariant derivatives are given by

$$
\nabla_{a}=e^{-\Omega^{+}} e^{-\boldsymbol{\Omega}^{+}} D_{a} e^{\boldsymbol{\Omega}^{+}} e^{\Omega^{+}} ; \quad \bar{\nabla}_{\dot{a}}=e^{\Omega} e^{\boldsymbol{\Omega}} \bar{D}_{\dot{a}} e^{-\boldsymbol{\Omega}} e^{-\Omega} .
$$

The subscript $A d j$ points out that they act on superfields in the adjoint representation. In particular, this implies that

$$
\left(f_{0}+f_{1} V+f_{2} V^{2}+\ldots\right)_{A d j} X \equiv f_{0} X+f_{1}[V, X]+f_{2}[V,[V, X]]+\ldots
$$


The functions $R(x)$ and $F(x)$ satisfy the conditions $R(0)=1, F(0)=1$ and have polynomial growth in the limit $x \rightarrow \infty$.

One can verify that the expression (2.17) is invariant both under the background gauge transformations (2.11) and under the quantum gauge transformations (2.12). Taking into account that

$$
-\frac{\bar{D}^{2} D^{2}}{16 \Lambda^{2}} \phi=\frac{\partial^{2}}{\Lambda^{2}} \phi
$$

for an arbitrary chiral superfield $\phi$, we see that in the lowest order in the (background and quantum) gauge superfields the regulators give $R\left(\partial^{2} / \Lambda^{2}\right)-1$ and $F\left(\partial^{2} / \Lambda^{2}\right)-1$. That is why eq. (2.17) is really a supersymmetric higher derivative term. After adding $S_{\Lambda}$ to the classical action $S$ we obtain the regularized action

$$
S_{\mathrm{reg}}=S+S_{\Lambda}
$$

It is convenient to choose the gauge fixing term in the form

$$
S_{\mathrm{gf}}=-\frac{1}{16 \xi_{0} e_{0}^{2}} \operatorname{tr} \int d^{4} x d^{4} \theta \nabla^{2} V K\left(-\frac{\bar{\nabla}^{2} \nabla^{2}}{16 \Lambda^{2}}\right)_{A d j} \bar{\nabla}^{2} V,
$$

because it does not break the background gauge invariance (2.11). Here the function $K(x)$ by construction satisfies the conditions $K(0)=1, K(\infty)=\infty$, and $\xi_{0}$ is a constant. In this case the standard gauge fixing procedure leads to the following actions for the FaddeevPopov and Nielsen-Kallosh ghosts:

$$
\begin{aligned}
& S_{\mathrm{FP}}=\frac{1}{e_{0}^{2}} \operatorname{tr} \int d^{4} x d^{4} \theta\left(e^{\boldsymbol{\Omega}} \bar{c} e^{-\boldsymbol{\Omega}}+e^{-\boldsymbol{\Omega}^{+}} \bar{c}^{+} e^{\boldsymbol{\Omega}^{+}}\right) \\
& \times\left\{\left(\frac{V}{1-e^{2 V}}\right)_{A d j}\left(e^{-\boldsymbol{\Omega}^{+}} c^{+} e^{\boldsymbol{\Omega}^{+}}\right)+\left(\frac{V}{1-e^{-2 V}}\right)_{A d j}\left(e^{\boldsymbol{\Omega}} c e^{-\boldsymbol{\Omega}}\right)\right\} ; \\
& S_{\mathrm{NK}}=\frac{1}{2 e_{0}^{2}} \operatorname{tr} \int d^{4} x d^{4} \theta b^{+}\left[e^{\boldsymbol{\Omega}^{+}} K\left(-\frac{\overline{\boldsymbol{\nabla}}^{2} \boldsymbol{\nabla}^{2}}{16 \Lambda^{2}}\right) e^{\boldsymbol{\Omega}}\right]_{A d j} b .
\end{aligned}
$$

The ghost superfields $c=e_{0} c^{A} t^{A}, \bar{c}=e_{0} \bar{c}^{A} t^{A}, b=e_{0} b^{A} t^{A}$ are anticommuting and chiral. As usual, the Nielsen-Kallosh ghosts interact only with the background gauge superfield $\boldsymbol{V}$ and, therefore, non-trivially contribute only in the one-loop approximation.

Introducing an auxiliary (commuting) chiral superfield $f$ it is also convenient to present the gauge fixing term in the form

$$
\begin{array}{r}
S_{\mathrm{gf}}=\frac{1}{e_{0}^{2}} \operatorname{tr} \int d^{4} x d^{4} \theta\left(16 \xi_{0} f^{+}\left[e^{\boldsymbol{\Omega}^{+}} K^{-1}\left(-\frac{\overline{\boldsymbol{\nabla}}^{2} \boldsymbol{\nabla}^{2}}{16 \Lambda^{2}}\right) e^{\boldsymbol{\Omega}}\right]_{\text {Adj }} f\right. \\
\left.+e^{\boldsymbol{\Omega}} f e^{-\boldsymbol{\Omega}} \boldsymbol{\nabla}^{2} V+e^{-\boldsymbol{\Omega}^{+}} f^{+} e^{\boldsymbol{\Omega}^{+}} \overline{\boldsymbol{\nabla}}^{2} V\right) .
\end{array}
$$

One can easily verify that the sum

$$
S+S_{\Lambda}+S_{\mathrm{gf}}+S_{\mathrm{FP}}+S_{\mathrm{NK}}
$$


which is obtained after the gauge fixing procedure, is invariant under the background gauge transformations (2.11), under which $f$ and ghost superfields should be transformed as

$$
f \rightarrow e^{A} f e^{-A}=\left(e^{A}\right)_{A d j} f ; \quad c \rightarrow\left(e^{A}\right)_{A d j} c ; \quad \bar{c} \rightarrow\left(e^{A}\right)_{A d j} \bar{c} ; \quad b \rightarrow\left(e^{A}\right)_{\text {Adj }} b .
$$

However, it is evident that the action (2.26) is not invariant under the quantum gauge transformation (2.12). Instead of this invariance the total action becomes invariant under the BRST transformations

$$
\begin{aligned}
& \delta V=-\varepsilon\left\{\left(\frac{V}{1-e^{2 V}}\right)_{A d j}\left(e^{-\boldsymbol{\Omega}^{+}} c^{+} e^{\boldsymbol{\Omega}^{+}}\right)+\left(\frac{V}{1-e^{-2 V}}\right)_{A d j}\left(e^{\boldsymbol{\Omega}} c e^{-\boldsymbol{\Omega}}\right)\right\} ; \quad \delta \phi=\varepsilon c \phi ; \\
& \delta \bar{c}=\varepsilon \bar{D}^{2}\left(e^{-2 \boldsymbol{V}} f^{+} e^{2 \boldsymbol{V}}\right) ; \quad \delta \bar{c}^{+}=\varepsilon D^{2}\left(e^{2 \boldsymbol{V}} f e^{-2 \boldsymbol{V}}\right) ; \quad \delta c=\varepsilon c^{2} ; \quad \delta c^{+}=\varepsilon\left(c^{+}\right)^{2} ; \\
& \delta f=\delta f^{+}=0 ; \quad \delta b=\delta b^{+}=0 ; \quad \delta \boldsymbol{\Omega}=\delta \boldsymbol{\Omega}^{+}=0,
\end{aligned}
$$

where $\varepsilon \neq \varepsilon(x)$ is an anticommuting real scalar parameter. Two first of these equations are equivalent to the equations (2.12) in which the parameter $\mathcal{A}$ is given by the expression

$$
\mathcal{A}=\varepsilon e^{\boldsymbol{\Omega}} c e^{-\boldsymbol{\Omega}} ; \quad \mathcal{A}^{+}=-\varepsilon e^{-\boldsymbol{\Omega}^{+}} c^{+} e^{\boldsymbol{\Omega}^{+}} .
$$

This allows us to verify the nilpotency of the BRST transformations. Really, the equalities $\delta_{1} \delta_{2} c=0$ and $\delta_{1} \delta_{2} \bar{c}=0$ are evident, and

$$
\delta_{1} \delta_{2} e^{2 V}=\delta_{1}\left(\varepsilon_{2} e^{-\boldsymbol{\Omega}^{+}} c^{+} e^{\boldsymbol{\Omega}^{+}} e^{2 V}-e^{2 V} \varepsilon_{2} e^{\boldsymbol{\Omega}} c e^{-\boldsymbol{\Omega}}\right)=0 .
$$

As a consequence, we obtain that $\delta_{1} \delta_{2} V=0$ and see that the BRST transformations are nilpotent. Writing the gauge fixing term in the form (2.25) and using this property, one can easily verify the BRST invariance of the action (2.26).

By introducing the higher derivative term one regularizes divergences beyond the oneloop approximation [71]. In order to get rid of the remaining one-loop divergences, it is necessary to insert the Pauli-Villars determinants into the generating functional [72]. Due to the absence of quadratic divergences in supersymmetric theories it is possible to use the following Pauli-Villars determinants:

$$
\begin{aligned}
Z[\boldsymbol{V}, \text { Sources }]=\int D V D \phi D b D \bar{c} D c \operatorname{Det}\left(P V, M_{\Phi}\right) \operatorname{Det}\left(P V, M_{\varphi}\right)^{-1} \\
\quad \times \exp \left(i S+i S_{\Lambda}+i S_{\mathrm{gf}}+i S_{\mathrm{FP}}+i S_{\mathrm{NK}}+i S_{\text {source }}\right),
\end{aligned}
$$

where

$$
\operatorname{Det}\left(P V, M_{\Phi}\right)=\int D \Phi \exp \left(i S_{\Phi}\right) ; \quad \operatorname{Det}\left(P V, M_{\varphi}\right)^{-1}=\int D \varphi \exp \left(i S_{\varphi}\right) .
$$

Here $\Phi_{i}$ is an anticommuting superfield in the same representation as $\phi_{i}$, three commuting superfields $\varphi_{f}$ lie in the adjoint representation of the gauge group, and the actions for the 
Pauli-Villars superfields are given by

$$
\begin{aligned}
S_{\Phi}= & \frac{1}{4} \int d^{4} x d^{4} \theta \Phi^{* i}\left[e^{\boldsymbol{\Omega}^{+}} e^{\Omega^{+}} F\left(-\frac{\bar{\nabla}^{2} \nabla^{2}}{16 \Lambda^{2}}\right) e^{\Omega} e^{\boldsymbol{\Omega}}\right]_{i}^{j} \Phi_{j}+\left(\frac{1}{4} \int d^{4} x d^{2} \theta\left(M_{\Phi}\right)^{i j} \Phi_{i} \Phi_{j}+\text { c.c. }\right) \\
S_{\varphi}= & \frac{1}{2 e_{0}^{2}} \operatorname{tr} \int d^{4} x d^{4} \theta\left(\varphi_{1}^{+}\left[e^{\boldsymbol{\Omega}^{+}} e^{\Omega^{+}} R\left(-\frac{\bar{\nabla}^{2} \nabla^{2}}{16 \Lambda^{2}}\right) e^{\Omega} e^{\boldsymbol{\Omega}}\right]_{A d j} \varphi_{1}+\varphi_{2}^{+}\left[e^{\boldsymbol{\Omega}^{+}} e^{2 V} e^{\boldsymbol{\Omega}}\right]_{A d j} \varphi_{2}\right. \\
& \left.+\varphi_{3}^{+}\left[e^{\boldsymbol{\Omega}^{+}} e^{2 V} e^{\boldsymbol{\Omega}}\right]_{A d j} \varphi_{3}\right)+\frac{1}{2 e_{0}^{2}} \operatorname{tr}\left(\int d^{4} x d^{2} \theta M_{\varphi}\left(\varphi_{1}^{2}+\varphi_{2}^{2}+\varphi_{3}^{2}\right)+\text { c.c. }\right) .
\end{aligned}
$$

We will also assume that

$$
\left(M_{\Phi}\right)^{j i}\left(M_{\Phi}^{*}\right)_{k j}=M_{\Phi}^{2} \delta_{k}^{i} .
$$

Below we will see that the chiral scalar superfields $\varphi_{f}$ introduced in this way exactly cancel the one-loop (sub)divergences introduced by loops of the gauge superfield and ghosts.

The actions (2.33) are also BRST invariant, because they are evidently invariant under the quantum gauge transformations (2.12) if

$$
\Phi \rightarrow e^{-\boldsymbol{\Omega}} e^{\mathcal{A}} e^{\boldsymbol{\Omega}} \Phi ; \quad \varphi_{f} \rightarrow e^{-\boldsymbol{\Omega}} e^{\mathcal{A}} e^{\boldsymbol{\Omega}} \varphi_{f} e^{-\boldsymbol{\Omega}} e^{-\mathcal{A}} e^{\boldsymbol{\Omega}}=\left(e^{-\boldsymbol{\Omega}} e^{\mathcal{A}} e^{\boldsymbol{\Omega}}\right)_{A d j} \varphi_{f} .
$$

For $\mathcal{A}$ given by eq. (2.29) we obtain the BRST invariance.

Thus, the final expression for the generating functional can be written as

$$
Z[\boldsymbol{V}, \text { Sources }]=\int D \mu \exp \left(i S_{\text {total }}+i S_{\text {source }}\right),
$$

where $\int D \mu$ denotes the integration measure which includes integration over all superfields of the theory, and the total action

$$
S_{\text {total }}=S+S_{\Lambda}+S_{\mathrm{gf}}+S_{\mathrm{FP}}+S_{\mathrm{NK}}+S_{\Phi}+S_{\varphi}
$$

is invariant under the above described BRST transformations. The generating functional for the connected Green functions is given by

$$
W[\boldsymbol{V}, \text { Sources }]=-i \ln Z[\boldsymbol{V}, \text { Sources }],
$$

and the effective action $\Gamma[\boldsymbol{V}$, Fields $]$ is defined in the standard way by using the Legendre transformation.

\subsection{Renormalization}

It is well known [73-76] that the considered supersymmetric theory is renormalizable, so that the divergences can be absorbed into redefinitions of the coupling constants and (super)fields. Taking into account that the superpotential does not receive the divergent quantum corrections according to the non-renormalization theorem [1] we can make the following renormalization:

$$
\begin{aligned}
\frac{1}{\alpha_{0}} & =\frac{Z_{\alpha}}{\alpha} ; \quad \frac{1}{\xi_{0}}=\frac{Z_{\xi}}{\xi} ; \quad V=V_{R} ; \quad V=Z_{V} Z_{\alpha}^{-1 / 2} V_{R} ; \quad \bar{c} c=Z_{c} Z_{\alpha}^{-1} \bar{c}_{R} c_{R} ; \\
\phi_{i} & =\left(\sqrt{Z_{\phi}}\right)_{i}{ }^{j}\left(\phi_{R}\right)_{j} ; \quad \lambda^{i j k}=\lambda_{0}^{m n p}\left(\sqrt{Z_{\phi}}\right)_{m}{ }^{i}\left(\sqrt{Z_{\phi}}\right)_{n}{ }^{j}\left(\sqrt{Z_{\phi}}\right)_{p}{ }^{k} ; \quad b=\sqrt{Z_{b}} b_{R},
\end{aligned}
$$


where $\alpha$ and $\lambda^{i j k}$ are the renormalized coupling constant and Yukawa couplings, respectively, and the renormalized superfields are denoted by the subscript $R$. We also take into account that the background gauge superfield is not renormalized due to the unbroken background gauge symmetry (2.11). From the definitions (2.39) we see that $Z_{c}$ denotes the renormalization constant for the Faddeev-Popov ghosts; $Z_{V}$ is the renormalization constant for the quantum gauge superfield, and $Z_{\alpha}$ encodes the charge renormalization.

Because the Nielsen-Kallosh action is not renormalized, the renormalization constants satisfy the relation $Z_{\alpha} Z_{b}=1$. Similarly, taking into account that the two-point Green function of the quantum gauge superfield is transversal due to the Slavnov-Taylor identity $[55,56]$, we obtain the relation $Z_{\xi} Z_{V}^{2}=1$.

The renormalization constants $Z_{\alpha}$ and $Z_{\phi}$ can be found by calculating the two-point Green functions of the background gauge superfield $\boldsymbol{V}$ and the matter superfields, respectively. Due to the background gauge invariance (2.11) the corresponding part of the effective action can be written in the form

$$
\begin{aligned}
\Gamma_{\boldsymbol{V}, \phi}^{(2)}=-\frac{1}{8 \pi} \operatorname{tr} \int \frac{d^{4} p}{(2 \pi)^{4}} & d^{4} \theta \boldsymbol{V}(\theta,-p) \partial^{2} \Pi_{1 / 2} \boldsymbol{V}(\theta, p) d^{-1}\left(\alpha_{0}, \lambda_{0}, \Lambda / p\right) \\
& +\frac{1}{4} \int \frac{d^{4} p}{(2 \pi)^{4}} d^{4} \theta \phi^{* i}(\theta,-p) \phi_{j}(\theta, p)\left(G_{\phi}\right)_{i}{ }^{j}\left(\alpha_{0}, \lambda_{0}, \Lambda / p\right),
\end{aligned}
$$

where $\partial^{2} \Pi_{1 / 2}=-D^{a} \bar{D}^{2} D_{a} / 8$ denotes the supersymmetric transversal projection operator. The functions $d^{-1}$ and $\left(G_{\phi}\right)_{i}{ }^{j}$ are, in general, divergent in the limit $\Lambda \rightarrow \infty$. The renormalized coupling constant $\alpha\left(\alpha_{0}, \lambda_{0}, \Lambda / \mu\right)$ and the renormalization constant $\left(Z_{\phi}\right)_{i}{ }^{j}\left(\alpha_{0}, \lambda_{0}, \Lambda / \mu\right)$, where $\mu$ is a renormalization point, are defined by requiring finiteness of the expressions

$$
\begin{aligned}
& d^{-1}\left(\alpha_{0}(\alpha, \lambda, \Lambda / \mu), \lambda_{0}(\alpha, \lambda, \Lambda / \mu), \Lambda / p\right) \\
& \left(Z_{\phi}\right)_{i}{ }^{j}\left(G_{\phi}\right)_{j}{ }^{k}\left(\alpha_{0}(\alpha, \lambda, \Lambda / \mu), \lambda_{0}(\alpha, \lambda, \Lambda / \mu), \Lambda / p\right)
\end{aligned}
$$

(considered as functions of $\alpha, \lambda, \mu / p$ and $\Lambda / p)$ in the limit $\Lambda \rightarrow \infty$. Then the renormalization constant $Z_{\alpha}$ is obtained from the equation

$$
Z_{\alpha}=\frac{\alpha}{\alpha_{0}} \text {. }
$$

In order to find the remaining renormalization constants $Z_{V}$ and $Z_{c}$ we consider the two-point Green functions of the quantum gauge superfield and ghosts. The Slavnov-Taylor identity ensures that all quantum corrections to the Green function of the quantum gauge superfield are transversal, so that

$$
\begin{aligned}
\Gamma_{V, c}^{(2)}-S_{\mathrm{gf}}^{(2)}= & -\frac{1}{2 e_{0}^{2}} \operatorname{tr} \int \frac{d^{4} p}{(2 \pi)^{4}} d^{4} \theta V(\theta,-p) \partial^{2} \Pi_{1 / 2} V(\theta, p) G_{V}\left(\alpha_{0}, \lambda_{0}, \Lambda / p\right) \\
& +\frac{1}{2 e_{0}^{2}} \operatorname{tr} \int \frac{d^{4} p}{(2 \pi)^{4}} d^{4} \theta\left(-\bar{c}(\theta,-p) c^{+}(\theta, p)+\bar{c}^{+}(\theta,-p) c(\theta, p)\right) G_{c}\left(\alpha_{0}, \lambda_{0}, \Lambda / p\right) .
\end{aligned}
$$


Then the renormalization constants $Z_{V}$ and $Z_{c}$ can be obtained by requiring finiteness of the functions

$$
\begin{aligned}
& Z_{V}^{2} G_{V}\left(\alpha_{0}(\alpha, \lambda, \Lambda / \mu), \lambda_{0}(\alpha, \lambda, \Lambda / \mu), \Lambda / p\right) \quad \text { and } \\
& Z_{c} G_{c}\left(\alpha_{0}(\alpha, \lambda, \Lambda / \mu), \lambda_{0}(\alpha, \lambda, \Lambda / \mu), \Lambda / p\right)
\end{aligned}
$$

in the limit $\Lambda \rightarrow \infty$, respectively.

\subsection{RG functions}

In this paper we consider the RG functions defined in terms of the bare coupling constant. In particular, the $\beta$-function is defined in terms of the bare coupling constants according to the prescription

$$
\beta\left(\alpha_{0}, \lambda_{0}\right)=\left.\frac{d \alpha_{0}(\alpha, \lambda, \Lambda / \mu)}{d \ln \Lambda}\right|_{\alpha, \lambda=\text { const }},
$$

and can be related to the renormalization constant $Z_{\alpha}$. Really, differentiating the first equation in (2.39) with respect to $\ln \Lambda$ we obtain

$$
\beta\left(\alpha_{0}, \lambda_{0}\right)=-\left.\alpha_{0} \frac{d \ln Z_{\alpha}}{d \ln \Lambda}\right|_{\alpha, \lambda=\text { const }} .
$$

For calculating this $\beta$-function it is convenient to consider the expression

$$
\left.\frac{d}{d \ln \Lambda}\left(d^{-1}\left(\alpha_{0}, \lambda_{0}, \Lambda / p\right)-\alpha_{0}^{-1}\right)\right|_{p=0}=-\frac{d \alpha_{0}^{-1}(\alpha, \lambda, \Lambda / \mu)}{d \ln \Lambda}=\frac{\beta\left(\alpha_{0}, \lambda_{0}\right)}{\alpha_{0}^{2}},
$$

in which the derivative with respect to $\ln \Lambda$ is calculated at fixed values of the renormalized coupling constant $\alpha$ and renormalized Yukawa constants $\lambda^{i j k}$ in the limit of the vanishing external momentum $p$.

The anomalous dimensions are defined in terms of the bare coupling constants by the equations

$$
\begin{gathered}
\left(\gamma_{\phi}\right)_{i}{ }^{j}\left(\alpha_{0}, \lambda_{0}\right) \equiv-\left.\frac{d \ln \left(Z_{\phi}\right)_{i}{ }^{j}(\alpha, \lambda, \Lambda / \mu)}{d \ln \Lambda}\right|_{\alpha, \lambda=\mathrm{const}}=\left.\frac{d \ln \left(G_{\phi}\right)_{i}{ }^{j}\left(\alpha_{0}, \lambda_{0}, \Lambda / p\right)}{d \ln \Lambda}\right|_{\alpha, \lambda=\mathrm{const} ; p=0} \\
\gamma_{V}\left(\alpha_{0}, \lambda_{0}\right) \equiv-\left.\frac{d \ln Z_{V}(\alpha, \lambda, \Lambda / \mu)}{d \ln \Lambda}\right|_{\alpha, \lambda=\mathrm{const}}=\left.\frac{1}{2} \cdot \frac{d \ln G_{V}\left(\alpha_{0}, \lambda_{0}, \Lambda / p\right)}{d \ln \Lambda}\right|_{\alpha, \lambda=\mathrm{const} ; p=0} ; \\
\gamma_{c}\left(\alpha_{0}, \lambda_{0}\right) \equiv-\left.\frac{d \ln Z_{c}(\alpha, \lambda, \Lambda / \mu)}{d \ln \Lambda}\right|_{\alpha, \lambda=\mathrm{const}}=\left.\frac{d \ln G_{c}\left(\alpha_{0}, \lambda_{0}, \Lambda / p\right)}{d \ln \Lambda}\right|_{\alpha, \lambda=\mathrm{const} ; p=0} \cdot \quad(2.48)
\end{gathered}
$$

It is known [36] that the RG functions defined in terms of the bare couplings depend on the regularization, but do not depend on the subtraction scheme for a fixed regularization.

\section{The RG functions in the one-loop approximation}

\subsection{One-loop $\beta$-function}

The two-point Green function of the background gauge superfield in the one-loop approximation is contributed by the diagrams presented in figure 1 . In these diagrams external 

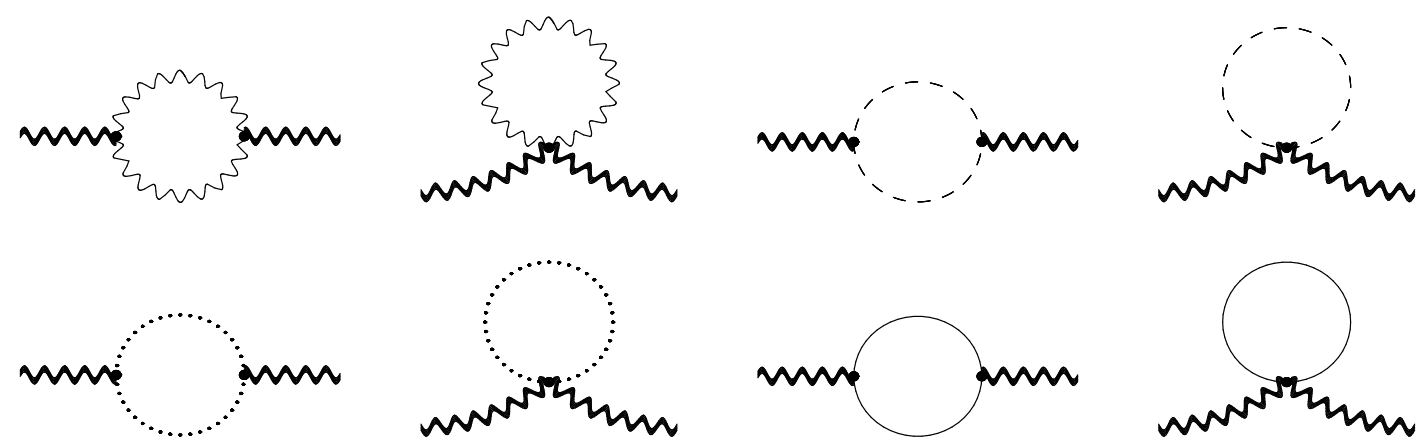

Figure 1. One-loop diagrams which contribute to the two-point Green function of the background superfield.

lines correspond to the superfield $\boldsymbol{V}$. The wavy internal lines denote propagators of the quantum gauge superfield $V$; the solid lines denote propagators of the matter superfields $\phi_{i}$ and of the Pauli-Villars superfields $\Phi_{i}$ and $\varphi_{f}$; the dashed lines denote propagators of the Faddeev-Popov ghosts; the dotted lines denote propagators of the Nielsen-Kallosh ghosts.

After calculating these diagrams we have obtained the following result:

$$
\frac{\beta\left(\alpha_{0}, \lambda_{0}\right)}{\alpha_{0}^{2}}=C_{2} I_{V}+T(R) I_{\phi}+O\left(\alpha_{0}, \lambda_{0}^{2}\right) .
$$

Here $I_{V}$ is the contribution of the quantum gauge superfield, the (Faddeev-Popov and Nielsen-Kallosh) ghosts, and the Pauli-Villars superfields $\varphi_{f} . I_{\phi}$ denotes the contribution of the matter superfield $\phi$ and the corresponding Pauli-Villars superfield $\Phi$. We have verified that both these integrals are integrals of double total derivatives independently of the concrete form of the functions $R$ and $F$ and have the following form:

$$
\begin{aligned}
I_{V}=\pi \int \frac{d^{4} q}{(2 \pi)^{4}} \frac{d}{d \ln \Lambda} \frac{\partial}{\partial q^{\mu}} \frac{\partial}{\partial q_{\mu}}\left[\frac{2}{q^{2}} \ln \left(\frac{R\left(q^{2} / \Lambda^{2}\right)}{K\left(q^{2} / \Lambda^{2}\right)}\right)\right. \\
\left.-\frac{2}{q^{2}} \ln \left(1+\frac{M_{\varphi}^{2}}{q^{2}}\right)-\frac{1}{q^{2}} \ln \left(\frac{q^{2} R^{2}\left(q^{2} / \Lambda^{2}\right)+M_{\varphi}^{2}}{q^{2} K^{2}\left(q^{2} / \Lambda^{2}\right)}\right)\right] \\
I_{\phi}=\pi \int \frac{d^{4} q}{(2 \pi)^{4}} \frac{d}{d \ln \Lambda} \frac{\partial}{\partial q^{\mu}} \frac{\partial}{\partial q_{\mu}}\left[\frac{1}{q^{2}} \ln \left(1+\frac{M_{\Phi}^{2}}{q^{2} F^{2}\left(q^{2} / \Lambda^{2}\right)}\right)\right]
\end{aligned}
$$

where $M_{\Phi}$ is defined by eq. (2.34). (The first term in the integral $I_{V}$ is the contribution of diagrams with the loop of the quantum gauge superfield $V$. The second term is a sum of diagrams with the loop of the Faddeev-Popov ghosts and the loop of the Pauli-Villars superfields $\varphi_{2}$ and $\varphi_{3}$. The last term corresponds to diagrams with the loop of the NielsenKallosh ghosts and the Pauli-Villars superfield $\varphi_{1}$.)

We see that all these integrals are integrals of double total derivatives in the momentum space. However, in general, they do not vanish, because of singularities of the integrands. Really, let $f\left(q^{2} / \Lambda^{2}\right)$ be a non-singular function with a rapid falloff at infinity. Then we consider the integral of the double total derivative

$$
I \equiv \int \frac{d^{4} q}{(2 \pi)^{4}} \frac{\partial}{\partial q^{\mu}} \frac{\partial}{\partial q_{\mu}}\left(\frac{1}{q^{2}} f\left(q^{2} / \Lambda^{2}\right)\right)
$$


This integral can be easily reduced to the integral of the $\delta$-function singularity:

$$
\begin{aligned}
I & =\int \frac{d^{4} q}{(2 \pi)^{4}} \frac{\partial}{\partial q^{\mu}}\left(-\frac{2 q^{\mu}}{q^{4}} f\left(q^{2} / \Lambda^{2}\right)+\frac{2 q^{\mu}}{q^{2} \Lambda^{2}} f^{\prime}\left(q^{2} / \Lambda^{2}\right)\right) \\
& =\int \frac{d^{4} q}{(2 \pi)^{4}} \frac{1}{q^{2}} \frac{d}{d q^{2}}\left(-4 f\left(q^{2} / \Lambda^{2}\right)+\frac{4 q^{2}}{\Lambda^{2}} f^{\prime}\left(q^{2} / \Lambda^{2}\right)\right) \\
& =\left.\frac{1}{4 \pi^{2}}\left(f\left(q^{2} / \Lambda^{2}\right)-\frac{q^{2}}{\Lambda^{2}} f^{\prime}\left(q^{2} / \Lambda^{2}\right)\right)\right|_{q=0}=\frac{1}{4 \pi^{2}} f(0)=4 \pi^{2} \int \frac{d^{4} q}{(2 \pi)^{4}} \delta^{4}(q) f\left(q^{2} / \Lambda^{2}\right)
\end{aligned}
$$

Using this result we calculate the integrals $I_{V}$ and $I_{\phi}$. For example, let us consider the integral $I_{\phi}$. First, we make the differentiation with respect to $\ln \Lambda$ taking into account that $M_{\Phi}$ is proportional to $\Lambda$ and, then, use eq. (3.5):

$$
\begin{aligned}
I_{\phi} & =\pi \int \frac{d^{4} q}{(2 \pi)^{4}} \frac{\partial}{\partial q^{\mu}} \frac{\partial}{\partial q_{\mu}}\left(\frac{2 M_{\Phi}^{2}}{q^{2}\left(q^{2} F^{2}\left(q^{2} / \Lambda^{2}\right)+M_{\Phi}^{2}\right)}+\frac{4 M_{\Phi}^{2} F^{\prime}\left(q^{2} / \Lambda^{2}\right)}{\Lambda^{2} F\left(q^{2} / \Lambda^{2}\right)\left(q^{2} F^{2}\left(q^{2} / \Lambda^{2}\right)+M_{\Phi}^{2}\right)}\right) \\
& =\int \frac{d^{4} q}{(2 \pi)^{4}} \delta^{4}(q) \frac{8 \pi^{3} M_{\Phi}^{2}}{\left(q^{2} F\left(q^{2} / \Lambda^{2}\right)+M_{\Phi}^{2}\right)}=\frac{1}{2 \pi} .
\end{aligned}
$$

Similarly, we obtain

$$
I_{V}=-\pi \int \frac{d^{4} q}{(2 \pi)^{4}} \frac{d}{d \ln \Lambda} \frac{\partial}{\partial q^{\mu}} \frac{\partial}{\partial q_{\mu}}\left[\frac{2}{q^{2}} \ln \left(1+\frac{M_{\varphi}^{2}}{q^{2}}\right)+\frac{1}{q^{2}} \ln \left(1+\frac{M_{\varphi}^{2}}{q^{2} R^{2}}\right)\right]=-\frac{3}{2 \pi} .
$$

Therefore, in the one-loop approximation the $\beta$-function (defined in terms of the bare coupling constant) is

$$
\beta=-\frac{\alpha_{0}^{2}}{2 \pi}\left(3 C_{2}-T(R)+O\left(\alpha_{0}, \lambda_{0}^{2}\right)\right) .
$$

Thus, we reobtain the standard expression for the one-loop $\beta$-function, which was first found in [77].

In the end of this section we note that eq. (3.1) can be also rewritten in the form

$$
\begin{aligned}
\frac{d \ln Z_{\alpha}}{d \ln \Lambda}=\pi \alpha_{0} \int & \frac{d^{4} q}{(2 \pi)^{4}} \frac{d}{d \ln \Lambda} \frac{\partial}{\partial q^{\mu}} \frac{\partial}{\partial q_{\mu}}\left[\frac{C_{2}}{q^{2}} \ln \left(1+\frac{M_{\varphi}^{2}}{q^{2} R^{2}}\right)\right. \\
& \left.+\frac{2 C_{2}}{q^{2}} \ln \left(1+\frac{M_{\varphi}^{2}}{q^{2}}\right)-\frac{T(R)}{q^{2}} \ln \left(1+\frac{M_{\Phi}^{2}}{q^{2} F^{2}}\right)\right]+O\left(\alpha_{0}^{2}, \alpha_{0} \lambda_{0}^{2}\right),
\end{aligned}
$$

which will be useful below.

\subsection{One-loop anomalous dimension of the matter superfields}

The one-loop anomalous dimension of the superfields $\phi_{i}$ for the considered theory is determined by the diagrams presented in figure 2. They give the following result for the anomalous dimension defined in terms of the bare coupling constant:

$$
\begin{aligned}
\left(\gamma_{\phi}\right)_{i}{ }^{j}\left(\alpha_{0}, \lambda_{0}\right)= & \int \frac{d^{4} k}{(2 \pi)^{4}} \frac{d}{d \ln \Lambda}\left(-C(R)_{i}{ }^{j} \frac{2 e^{2}}{k^{4} R\left(k^{2} / \Lambda^{2}\right)}+\lambda_{i m n}^{*} \lambda^{j m n} \frac{2}{k^{4} F^{2}\left(k^{2} / \Lambda^{2}\right)}\right) \\
& +O\left(\alpha^{2}, \alpha \lambda^{2}, \lambda^{4}\right) .
\end{aligned}
$$



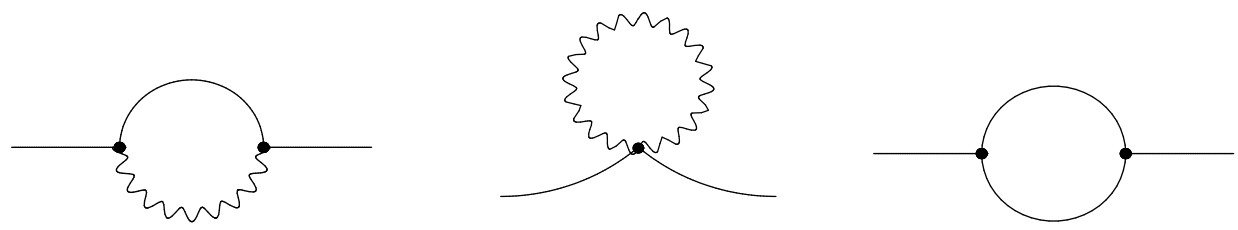

Figure 2. Diagrams contributing to the one-loop anomalous dimension of the matter superfield.

Due to the derivative with respect to $\ln \Lambda$ (which should be taken at fixed values of the renormalized coupling and Yukawa constants $e$ and $\lambda^{i j k}$, respectively) this integral is well defined. Taking into account that

$$
\frac{d}{d \ln \Lambda} f\left(k^{2} / \Lambda^{2}\right)=-\frac{d}{d \ln k} f\left(k^{2} / \Lambda^{2}\right)=-2 k^{2} \frac{d}{d k^{2}} f\left(k^{2} / \Lambda^{2}\right)
$$

and that for an arbitrary function $f$ with a sufficiently rapid falloff at infinity

$$
\int \frac{d^{4} k}{(2 \pi)^{4}} \frac{1}{k^{2}} \frac{d f}{d k^{2}}=-\frac{1}{16 \pi^{2}} f(0)
$$

we obtain

$$
\begin{aligned}
\left(\gamma_{\phi}\right)_{i}{ }^{j}\left(\alpha_{0}, \lambda_{0}\right)= & \int \frac{d^{4} k}{(2 \pi)^{4}} \frac{1}{k^{2}} \frac{d}{d k^{2}}\left(C(R)_{i}{ }^{j} \frac{4 e^{2}}{R\left(k^{2} / \Lambda^{2}\right)}-\lambda_{i m n}^{*} \lambda^{j m n} \frac{4}{F^{2}\left(k^{2} / \Lambda^{2}\right)}\right) \\
& +O\left(\alpha^{2}, \alpha \lambda^{2}, \lambda^{4}\right) \\
= & -C(R)_{i}{ }^{j} \frac{\alpha_{0}}{\pi}+\frac{1}{4 \pi^{2}} \lambda_{0 i m n}^{*} \lambda_{0}^{j m n}+O\left(\alpha_{0}^{2}, \alpha_{0} \lambda_{0}^{2}, \lambda_{0}^{4}\right) .
\end{aligned}
$$

Note that in the last equation the result is written in terms of the bare coupling constants $\alpha_{0}$ and $\lambda_{0}^{i j k}$, because we calculate the anomalous dimension defined in terms of the bare charges. Certainly, the expression (3.13) coincides with the well-known result. However, it is interesting to compare the integral (3.10) with the integrals which give the two-loop $\beta$-function with the considered regularization, as it was done in, e.g., [40].

\subsection{One-loop renormalization of the quantum gauge superfield}

From the two-point Green function of the quantum gauge superfield we can find the constant $Z_{V}^{2}$. In the one-loop approximation this Green function is contributed by the diagrams presented in figure 3. After calculating them in the limit of the vanishing external momentum we obtained

$$
\begin{aligned}
\frac{d \ln Z_{V}^{2}}{d \ln \Lambda}= & \pi \alpha_{0} \int \frac{d^{4} q}{(2 \pi)^{4}} \frac{d}{d \ln \Lambda}\left(\frac { \partial } { \partial q ^ { \mu } } \frac { \partial } { \partial q _ { \mu } } \left[\frac{C_{2}}{q^{2}} \ln \left(1+\frac{M_{\varphi}^{2}}{q^{2} R^{2}}\right)+\frac{2 C_{2}}{q^{2}} \ln \left(1+\frac{M_{\varphi}^{2}}{q^{2}}\right)\right.\right. \\
& \left.\left.-\frac{T(R)}{q^{2}} \ln \left(1+\frac{M_{\Phi}^{2}}{q^{2} F^{2}}\right)\right]+8 C_{2}\left(-\frac{1}{3 R q^{4}}+\frac{\xi_{0}}{3 K q^{4}}\right)+O\left(\alpha_{0}, \lambda_{0}^{2}\right)\right) .
\end{aligned}
$$

Comparing this equation with the expression $d \ln Z_{\alpha} / d \ln \Lambda$ given by eq. (3.9) we obtain

$$
\gamma_{V}=-4 \pi \alpha_{0} C_{2} \int \frac{d^{4} q}{(2 \pi)^{4}} \frac{d}{d \ln \Lambda}\left(-\frac{1}{3 R q^{4}}+\frac{\xi_{0}}{3 K q^{4}}\right)-\frac{1}{2} \cdot \frac{d \ln Z_{\alpha}}{d \ln \Lambda}+O\left(\alpha_{0}^{2}, \alpha_{0} \lambda_{0}^{2}\right)
$$



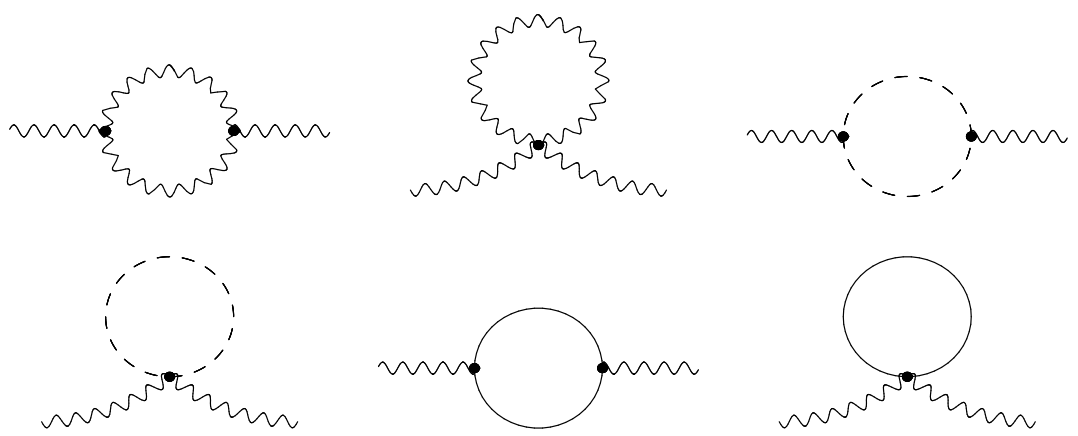

Figure 3. One-loop diagrams contributing to the two-point Green function of the quantum gauge superfield.

The integral in this expression can be easily calculated by using eq. (3.11):

$$
\begin{aligned}
\int \frac{d^{4} q}{(2 \pi)^{4}} \frac{d}{d \ln \Lambda}\left(-\frac{1}{3 R q^{4}}+\frac{\xi_{0}}{3 K q^{4}}\right) & =-\int \frac{d^{4} q}{(2 \pi)^{4}} \frac{2}{3 q^{2}} \frac{d}{d q^{2}}\left(-\frac{1}{R}+\frac{\xi_{0}}{K}\right) \\
& =-\frac{1}{24 \pi^{2}}\left(\frac{1}{R(0)}-\frac{\xi_{0}}{K(0)}\right)=-\frac{\left(1-\xi_{0}\right)}{24 \pi^{2}}
\end{aligned}
$$

so that finally we obtain

$$
\gamma_{V}=\frac{\alpha_{0} C_{2}\left(1-\xi_{0}\right)}{6 \pi}+\frac{\beta\left(\alpha_{0}, \lambda_{0}\right)}{2 \alpha_{0}}+O\left(\alpha_{0}^{2}, \alpha_{0} \lambda_{0}^{2}\right)
$$

\subsection{One-loop renormalization of the Faddeev-Popov ghosts}

In order to find the anomalous dimension of the Faddeev-Popov ghosts (defined in terms of the bare coupling constant) it is necessary to calculate the diagrams presented in figure 4 . It is convenient to write the result in the form

$$
\begin{aligned}
\gamma_{c} & =4 \pi \alpha_{0} C_{2} \int \frac{d^{4} q}{(2 \pi)^{4}} \frac{d}{d \ln \Lambda}\left(-\frac{1}{3 R q^{4}}+\frac{\xi_{0}}{3 K q^{4}}\right)+O\left(\alpha_{0}^{2}, \alpha_{0} \lambda_{0}^{2}\right) \\
& =-\frac{\alpha_{0} C_{2}\left(1-\xi_{0}\right)}{6 \pi}+O\left(\alpha_{0}^{2}, \alpha_{0} \lambda_{0}^{2}\right) .
\end{aligned}
$$

This implies that in the general gauge the two-point Green function of the Faddeev-Popov ghosts is divergent, and the higher covariant derivative regularization does regularize these divergences. However, comparing eq. (3.18) with eq. (3.15) (or eq. (3.17)) we see that in the one-loop approximation

$$
\frac{d}{d \ln \Lambda}\left(\ln Z_{c}+\ln Z_{V}-\frac{1}{2} \ln Z_{\alpha}\right)=0 .
$$

As a consequence, the vertices of the type $\bar{c} V c$ are finite. Possibly, this statement is valid in all loops if the regularization does not break the BRST invariance of the theory. Note that for some particular regularizations and gauge fixing conditions validity of eq. (3.19) in the one-loop approximation can also be seen from the results of refs. [54] for the pure $\mathcal{N}=1 \mathrm{SYM}$ theory and of refs. $[32,33]$ for the general $\mathcal{N}=2 \mathrm{SYM}$ theory with matter. 

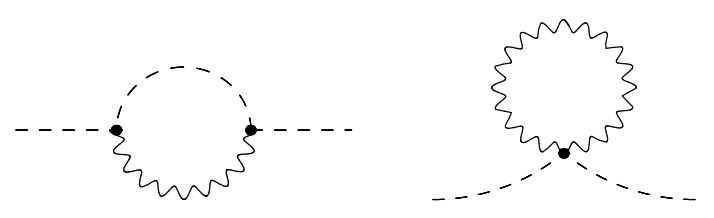

Figure 4. Diagrams contributing to the one-loop anomalous dimension of the Faddeev-Popov ghosts.

\section{Conclusion}

In this paper we consider a general $\mathcal{N}=1 \mathrm{SYM}$ theory with matter regularized by a very general version of the higher derivative regularization which does not break the BRST invariance and calculate all RG functions in the one-loop approximation. The considered version of the higher derivative regularization was not earlier used to obtain quantum corrections, because it leads to very complicated calculations. However, it does not break symmetries of the theory and seems to be very useful for the general derivation of the NSVZ $\beta$-function in the non-Abelian case by the direct summation of supergraphs. Making such a derivation one should consider the one-loop approximation separately, and this problem is addressed in this paper. In particular, we have demonstrated that all one-loop momentum integrals for the $\beta$-function are integrals of double total derivatives independently of the form of the higher derivative term. This seems to be a general feature of all supersymmetric theories. ${ }^{1}$ Certainly, the result of the calculation coincided with the well-known expression for the one-loop $\beta$-function in the supersymmetric case. Also we have obtained the momentum integrals defining the one-loop anomalous dimension, which also coincided with the well-known expression. In prospect, these integrals can be compared with integrals giving the two-loop $\beta$-function, which are related to them due to the existence of the NSVZ $\beta$-function. Also we obtained that the vertices $\bar{c} V c, \bar{c} V c^{+}, \bar{c}^{+} V c$, and $\bar{c}^{+} V c^{+}$ (containing two lines of the Faddeev-Popov ghosts and a single line of the quantum gauge superfield) are not renormalized in the considered approximation. Possibly, this feature is valid in an arbitrary order of the perturbation theory.

\section{Acknowledgments}

The authors are very grateful to D.S. Kolupaev for valuable discussions. The work of K.S. is supported by the RFBR grant No. 14-01-00695.

Open Access. This article is distributed under the terms of the Creative Commons Attribution License (CC-BY 4.0), which permits any use, distribution and reproduction in any medium, provided the original author(s) and source are credited.

\footnotetext{
${ }^{1}$ The similar structures for Abelian $\mathcal{N}=1$ supersymmetric theories regularized by the dimensional reduction were considered in [78].
} 


\section{References}

[1] M.T. Grisaru, W. Siegel and M. Roček, Improved Methods for Supergraphs, Nucl. Phys. B 159 (1979) 429 [INSPIRE].

[2] V.A. Novikov, M.A. Shifman, A.I. Vainshtein and V.I. Zakharov, Exact Gell-Mann-Low Function of Supersymmetric Yang-Mills Theories from Instanton Calculus, Nucl. Phys. B 229 (1983) 381 [INSPIRE].

[3] D.R.T. Jones, More on the Axial Anomaly in Supersymmetric Yang-Mills Theory, Phys. Lett. B 123 (1983) 45 [inSPIRE].

[4] V.A. Novikov, M.A. Shifman, A.I. Vainshtein and V.I. Zakharov, The beta function in supersymmetric gauge theories. Instantons versus traditional approach, Phys. Lett. B 166 (1986) 329 [Sov. J. Nucl. Phys. 43 (1986) 294] [Yad. Fiz. 43 (1986) 459] [InSPIRE].

[5] M.A. Shifman and A.I. Vainshtein, Solution of the Anomaly Puzzle in SUSY Gauge Theories and the Wilson Operator Expansion, Nucl. Phys. B 277 (1986) 456 [Sov. Phys. JETP 64 (1986) 428] [Zh. Eksp. Teor. Fiz. 91 (1986) 723] [INSPIRE].

[6] A.I. Vainshtein, V.I. Zakharov and M.A. Shifman, Gell-mann-low Function In Supersymmetric Electrodynamics, JETP Lett. 42 (1985) 224 [Pisma Zh. Eksp. Teor. Fiz. 42 (1985) 182] [INSPIRE].

[7] M.A. Shifman, A.I. Vainshtein and V.I. Zakharov, Exact Gell-mann-low Function In Supersymmetric Electrodynamics, Phys. Lett. B 166 (1986) 334 [INSPIRE].

[8] M.A. Shifman and A.I. Vainshtein, Instantons versus supersymmetry: Fifteen years later, in M.A. Shifman, ITEP lectures on particle physics and field theory. Volume 2, World Scientific Publishing Co Pte Ltd (1999), pp. 485-647, [hep-th/9902018] [INSPIRE].

[9] N. Arkani-Hamed and H. Murayama, Holomorphy, rescaling anomalies and exact $\beta$-functions in supersymmetric gauge theories, JHEP 06 (2000) 030 [hep-th/9707133] [INSPIRE].

[10] E. Kraus, C. Rupp and K. Sibold, Supersymmetric Yang-Mills theories with local coupling: The Supersymmetric gauge, Nucl. Phys. B 661 (2003) 83 [hep-th/0212064] [INSPIRE].

[11] L.V. Avdeev and O.V. Tarasov, The Three Loop $\beta$-function in the $N=1, N=2, N=4$ Supersymmetric Yang-Mills Theories, Phys. Lett. B 112 (1982) 356 [InSPIRE].

[12] I. Jack, D.R.T. Jones and C.G. North, $N=1$ supersymmetry and the three loop gauge -function, Phys. Lett. B 386 (1996) 138 [hep-ph/9606323] [INSPIRE].

[13] I. Jack, D.R.T. Jones and C.G. North, Scheme dependence and the NSVZ $\beta$-function, Nucl. Phys. B 486 (1997) 479 [hep-ph/9609325] [INSPIRE].

[14] R.V. Harlander, D.R.T. Jones, P. Kant, L. Mihaila and M. Steinhauser, Four-loop $\beta$-function and mass anomalous dimension in dimensional reduction, JHEP 12 (2006) 024 [hep-ph/0610206] [INSPIRE].

[15] I. Jack, D.R.T. Jones, P. Kant and L. Mihaila, The Four-loop DRED gauge $\beta$-function and fermion mass anomalous dimension for general gauge groups, JHEP 09 (2007) 058 [arXiv:0707.3055] [INSPIRE].

[16] L. Mihaila, Precision Calculations in Supersymmetric Theories, Adv. High Energy Phys. 2013 (2013) 607807 [arXiv: 1310.6178] [INSPIRE].

[17] W. Siegel, Supersymmetric Dimensional Regularization via Dimensional Reduction, Phys. Lett. B 84 (1979) 193 [INSPIRE]. 
[18] I. Jack, D.R.T. Jones and A. Pickering, The Connection between DRED and NSVZ, Phys. Lett. B 435 (1998) 61 [hep-ph/9805482] [INSPIRE].

[19] D. Kutasov and A. Schwimmer, Lagrange multipliers and couplings in supersymmetric field theory, Nucl. Phys. B 702 (2004) 369 [hep-th/0409029] [InSPIRE].

[20] A.L. Kataev and K.V. Stepanyantz, The NSVZ $\beta$-function in supersymmetric theories with different regularizations and renormalization prescriptions, Theor. Math. Phys. 181 (2014) 1531 [arXiv: 1405.7598] [INSPIRE].

[21] A.L. Kataev and K.V. Stepanyantz, Scheme independent consequence of the NSVZ relation for $N=1 S Q E D$ with $N_{f}$ flavors, Phys. Lett. B 730 (2014) 184 [arXiv:1311.0589] [INSPIRE].

[22] A.A. Slavnov, Invariant regularization of nonlinear chiral theories, Nucl. Phys. B 31 (1971) 301 [INSPIRE].

[23] A.A. Slavnov, Invariant regularization of gauge theories (in Russian), Teor. Mat. Fiz. 13 (1972) 174 [Theor. Math. Phys. 13 (1972) 1064] [InSPIRE].

[24] W. Siegel, Inconsistency of Supersymmetric Dimensional Regularization, Phys. Lett. B 94 (1980) 37 [INSPIRE].

[25] L.V. Avdeev, G.A. Chochia and A.A. Vladimirov, On the Scope of Supersymmetric Dimensional Regularization, Phys. Lett. B 105 (1981) 272 [INSPIRE].

[26] L.V. Avdeev and A.A. Vladimirov, Dimensional Regularization and Supersymmetry, Nucl. Phys. B 219 (1983) 262 [INSPIRE].

[27] L.V. Avdeev, Noninvariance of Regularization by Dimensional Reduction: An Explicit Example of Supersymmetry Breaking, Phys. Lett. B 117 (1982) 317 [INSPIRE].

[28] V.N. Velizhanin, Three-loop renormalization of the $N=1, N=2, N=4$ supersymmetric Yang-Mills theories, Nucl. Phys. B 818 (2009) 95 [arXiv: 0809.2509] [INSPIRE].

[29] V.K. Krivoshchekov, Invariant Regularizations for Supersymmetric Gauge Theories, Theor. Math. Phys. 36 (1978) 745 [Teor. Mat. Fiz. 36 (1978) 291] [InSPIRE].

[30] P.C. West, Higher Derivative Regulation of Supersymmetric Theories, Nucl. Phys. B 268 (1986) 113 [INSPIRE].

[31] V.K. Krivoshchekov, Invariant Regularization For $N=2$ Superfield Perturbation Theory, Phys. Lett. B 149 (1984) 128 [inSPIRE].

[32] I.L. Buchbinder and K.V. Stepanyantz, The higher derivative regularization and quantum corrections in $N=2$ supersymmetric theories, Nucl. Phys. B 883 (2014) 20 [arXiv: 1402.5309] [INSPIRE].

[33] I.L. Buchbinder, N.G. Pletnev and K.V. Stepanyantz, Manifestly $N=2$ supersymmetric regularization for $N=2$ supersymmetric field theories, Phys. Lett. B 751 (2015) 434 [arXiv: 1509.08055] [INSPIRE].

[34] K.V. Stepanyantz, Derivation of the exact NSVZ $\beta$-function in $N=1 S Q E D$, regularized by higher derivatives, by direct summation of Feynman diagrams, Nucl. Phys. B 852 (2011) 71 [arXiv: 1102.3772] [INSPIRE].

[35] K.V. Stepanyantz, The NSVZ $\beta$-function and the Schwinger-Dyson equations for $\mathcal{N}=1$ $S Q E D$ with $N_{f}$ flavors, regularized by higher derivatives, JHEP 08 (2014) 096 [arXiv: 1404.6717] [INSPIRE]. 
[36] A.L. Kataev and K.V. Stepanyantz, NSVZ scheme with the higher derivative regularization for $\mathcal{N}=1$ SQED, Nucl. Phys. B 875 (2013) 459 [arXiv: 1305.7094] [INSPIRE].

[37] N.N. Bogolyubov and D.V. Shirkov, Introduction To The Theory Of Quantized Fields, Intersci. Monogr. Phys. Astron. 3 (1959) 1 [INSPIRE].

[38] A.A. Soloshenko and K.V. Stepanyantz, Three loop $\beta$-function for $N=1$ supersymmetric electrodynamics, regularized by higher derivatives, Theor. Math. Phys. 140 (2004) 1264 [Teor. Mat. Fiz. 140 (2004) 430] [hep-th/0304083] [INSPIRE].

[39] A.V. Smilga and A. Vainshtein, Background field calculations and nonrenormalization theorems in 4-D supersymmetric gauge theories and their low-dimensional descendants, Nucl. Phys. B 704 (2005) 445 [hep-th/0405142] [INSPIRE].

[40] A.E. Kazantsev and K.V. Stepanyantz, Relation between two-point Green's functions of $\mathcal{N}=1 S Q E D$ with $N_{f}$ flavors, regularized by higher derivatives, in the three-loop approximation, J. Exp. Theor. Phys. 120 (2015) 618 [arXiv:1410.1133] [INSPIRE].

[41] S.L. Adler, Some simple vacuum-polarization phenomenology: $e^{+} e^{-} \rightarrow$ hadrons; the muonic-atom x-ray discrepancy and $g_{\mu}-2$, Phys. Rev. D 10 (1974) 3714 [INSPIRE].

[42] M. Shifman and K.V. Stepanyantz, Exact Adler Function in Supersymmetric QCD, Phys. Rev. Lett. 114 (2015) 051601 [arXiv:1412.3382] [INSPIRE].

[43] M. Shifman and K.V. Stepanyantz, Derivation of the exact expression for the $D$ function in $N=1$ SQCD, Phys. Rev. D 91 (2015) 105008 [arXiv:1502.06655] [InSPIRE].

[44] A.B. Pimenov, E.S. Shevtsova and K.V. Stepanyantz, Calculation of two-loop $\beta$-function for general $N=1$ supersymmetric Yang-Mills theory with the higher covariant derivative regularization, Phys. Lett. B 686 (2010) 293 [arXiv:0912.5191] [INSPIRE].

[45] K.V. Stepanyantz, Higher covariant derivative regularization for calculations in supersymmetric theories, Proc. Steklov Inst. Math. 272 (2011) 256.

[46] K.V. Stepanyantz, Factorization of integrals defining the two-loop $\beta$-function for the general renormalizable $N=1 S Y M$ theory, regularized by the higher covariant derivatives, into integrals of double total derivatives, arXiv:1108.1491 [INSPIRE].

[47] K.V. Stepanyantz, Derivation of the exact NSVZ $\beta$-function in $N=1$ SQED regularized by higher derivatives by summation of Feynman diagrams, J. Phys. Conf. Ser. 343 (2012) 012115 [INSPIRE].

[48] K.V. Stepanyantz, Multiloop calculations in supersymmetric theories with the higher covariant derivative regularization, J. Phys. Conf. Ser. 368 (2012) 012052 [arXiv: 1203.5525] [INSPIRE].

[49] C. Becchi, A. Rouet and R. Stora, Renormalization of the Abelian Higgs-Kibble Model, Commun. Math. Phys. 42 (1975) 127 [inSPIRE].

[50] I.V. Tyutin, Gauge Invariance in Field Theory and Statistical Physics in Operator Formalism, LEBEDEV-75-39 [arXiv:0812.0580] [INSPIRE].

[51] A.A. Slavnov, Universal gauge invariant renormalization, Phys. Lett. B 518 (2001) 195 [INSPIRE].

[52] A.A. Slavnov, Regularization-independent gauge-invariant renormalization of the Yang-Mills theory, Theor. Math. Phys. 130 (2002) 1 [Teor. Mat. Fiz. 130 (2002) 3] [InSPIRE]. 
[53] A.A. Slavnov and K.V. Stepanyantz, Universal invariant renormalization for supersymmetric theories, Theor. Math. Phys. 135 (2003) 673 [Teor. Mat. Fiz. 135 (2003) 265] [hep-th/0208006] [INSPIRE].

[54] A.A. Slavnov and K.V. Stepanyantz, Universal invariant renormalization of supersymmetric Yang-Mills theory, Theor. Math. Phys. 139 (2004) 599 [Teor. Mat. Fiz. 139 (2004) 179] [hep-th/0305128] [INSPIRE].

[55] J.C. Taylor, Ward Identities and Charge Renormalization of the Yang-Mills Field, Nucl. Phys. B 33 (1971) 436 [INSPIRE].

[56] A.A. Slavnov, Ward Identities in Gauge Theories, Theor. Math. Phys. 10 (1972) 99 [Teor. Mat. Fiz. 10 (1972) 153] [INSPIRE].

[57] P.I. Pronin and K.V. Stepanyantz, One loop counterterms for higher derivative regularized Lagrangians, Phys. Lett. B 414 (1997) 117 [hep-th/9707008] [INSPIRE].

[58] C.P. Martin and F. Ruiz Ruiz, Higher covariant derivative Pauli-Villars regularization does not lead to a consistent QCD, Nucl. Phys. B 436 (1995) 545 [hep-th/9410223] [InSPIRE].

[59] D.J. Gross and F. Wilczek, Ultraviolet Behavior of Nonabelian Gauge Theories, Phys. Rev. Lett. 30 (1973) 1343 [INSPIRE].

[60] H.D. Politzer, Reliable Perturbative Results for Strong Interactions?, Phys. Rev. Lett. 30 (1973) 1346 [INSPIRE].

[61] M. Asorey and F. Falceto, On the consistency of the regularization of gauge theories by high covariant derivatives, Phys. Rev. D 54 (1996) 5290 [hep-th/9502025] [InSPIRE].

[62] T.D. Bakeyev and A.A. Slavnov, Higher covariant derivative regularization revisited, Mod. Phys. Lett. A 11 (1996) 1539 [hep-th/9601092] [INSPIRE].

[63] R. Turcati and M.J. Neves, Complex-mass shell renormalization of the higher-derivative electrodynamics, arXiv:1601.07218 [INSPIRE].

[64] H.G. Fargnoli, B. Hiller, A.P.B. Scarpelli, M. Sampaio and M.C. Nemes, Regularization Independent Analysis of the Origin of Two Loop Contributions to $N=1$ Super Yang-Mills B-function, Eur. Phys. J. C 71 (2011) 1633 [arXiv:1009.2976] [INSPIRE].

[65] A.L. Cherchiglia, M. Sampaio, B. Hiller and A.P.B. Scarpelli, Subtleties in the $\beta$-function calculation of $N=1$ supersymmetric gauge theories, Eur. Phys. J. C 76 (2016) 47 [arXiv: 1508.05421] [INSPIRE].

[66] P.C. West, Introduction to supersymmetry and supergravity, World Scientific, Singapore (1990).

[67] I.L. Buchbinder and S.M. Kuzenko, Ideas and methods of supersymmetry and supergravity: Or a walk through superspace, IOP Publishing, Bristol U.K. (1998).

[68] B.S. DeWitt, Dynamical theory of groups and fields, Conf. Proc. C 630701 (1964) 585 [Les Houches Lect. Notes 13 (1964) 585] [INSPIRE].

[69] L.F. Abbott, The Background Field Method Beyond One Loop, Nucl. Phys. B 185 (1981) 189 [INSPIRE].

[70] L.F. Abbott, Introduction to the Background Field Method, Acta Phys. Polon. B 13 (1982) 33 [INSPIRE].

[71] L.D. Faddeev and A.A. Slavnov, Gauge Fields. Introduction To Quantum Theory, Front. Phys. 50 (1980) 1 [Front. Phys. 83 (1990) 1] [INSPIRE]. 
[72] A.A. Slavnov, The Pauli-Villars Regularization for Nonabelian Gauge Theories (in Russian), Teor. Mat. Fiz. 33 (1977) 210 [Theor. Math. Phys. 33 (1977) 977] [InSPIRE].

[73] A.A. Slavnov, Renormalization of Supersymmetric Gauge Theories. 2. Nonabelian Case, Nucl. Phys. B 97 (1975) 155 [inSPIRE].

[74] S. Ferrara and O. Piguet, Perturbation Theory and Renormalization of Supersymmetric Yang-Mills Theories, Nucl. Phys. B 93 (1975) 261 [inSPIRE].

[75] O. Piguet and A. Rouet, Supersymmetric BPHZ Renormalization. 2. Supersymmetric Extension of Pure Yang-Mills Model, Nucl. Phys. B 108 (1976) 265 [INSPIRE].

[76] O. Piguet and K. Sibold, Renormalization of $N=1$ Supersymmetrical Yang-Mills Theories. 2. The Radiative Corrections, Nucl. Phys. B 197 (1982) 272 [InSPIRE].

[77] S. Ferrara and B. Zumino, Supergauge Invariant Yang-Mills Theories, Nucl. Phys. B 79 (1974) 413 [INSPIRE].

[78] S.S. Aleshin, A.L. Kataev and K.V. Stepanyantz, Structure of three-loop contributions to the $\beta$-function of $N=1 S Q E D$ with $N_{f}$ flavors, regularized by the dimensional reduction, JETP Lett. 103 (2016) 77 [Pisma Zh. Eksp. Teor. Fiz. 130 (2016) 83] [arXiv:1511.05675] [INSPIRE]. 